\title{
ASPECTOS HISTOMORFOLÓGICOS E RESPIRATÓRIOS EM MODELO DE LESÃO PULMONAR AGUDA POR SEPSE EM RATOS TRATADOS COM PENTOXIFILINA
}

\author{
Wagner Rogerio Souza de Oliveira ${ }^{1}$, Sâmia Santos Cavassani², Carla Cristina Maganhin ${ }^{3}$, Adriana Aparecida Ferraz Carbonel ${ }^{4}$, Manuel Jesus Simöes ${ }^{5}$, Ricardo \\ Santos Simöes ${ }^{6}$, Virginia Berlanga Campos Junqueira ${ }^{7}$, Itamar Souza de Oliveira-Júnior*8 \\ Trabalho realizado na Universidade Federal de São Paulo - Escola Paulista de Medicina, S.Paulo, SP
}

*Correspondência:

Rua Pedro de Toledo, 781

- 110. andar - Frente

Vila Clementino

São Paulo - SP

CEP 04039-032

\begin{abstract}
RESUMO
Овј втіvo. Analisar as alterações histomorfológicas e respiratórias em modelo de lesão pulmonar aguda por sepse em ratos tratados com pentoxifilina.

Métodos. Foram utilizados 15 ratos adultos distribuídos em três grupos ( $n=5$, por grupo), assim constituídos: GC - receberam apenas ventilação mecânica; GS - Animais sépticos tratados com solução salina e mecanicamente ventilados; GS+PTX - Animais sépticos, com infusão de pentoxifilina e mecanicamente ventilados. Todos os animais foram ventilados por um período de 180 minutos. Ao final deste período, foram avaliadas variáveis gasométricas (gasometria arterial), gravimétricas (relação peso úmido/peso seco), concentração de proteínas totais no lavado broncoalveolar e histomorfométricas (espessura dos septos alveolares). Os dados obtidos foram submetidos a análise estatística $(P<0,05)$ Resultados. A pressão parcial de oxigênio ao final do experimento mostrou-se elevada no grupo GS+PTX $(460,0 \pm 38,2 \mathrm{mmHg})$ em relação ao grupo $\mathrm{GS}(336,0 \pm 14,6 \mathrm{mmHg})(P<0,05)$. No grupo GS, a concentração de proteínas no lavado broncoalveolar encontrou-se aumentada em relação aos demais grupos; no entanto, se mostrou atenuada após a administração de pentoxifilina. N otamos, pela morfologia em todos os grupos avaliados, vasodilatação nos septos alveolares e no grupo $\mathrm{S}$ alguns alvéolos apresentaram-se repletos de macrófagos. Estes aspectos foram atenuados no GS+PTX. A espessura dos septos alveolares mostrou uma significante redução no grupo GS+PTX quando comparado com o grupo GS $(\mathrm{P}<0,05)$.

ConcLusão. A pentoxifilina restabelece a oxigenação e reduz os efeitos deletérios do processo de sepse em associação à ventilação mecânica com baixo volume corrente.
\end{abstract}

Unitermos: Sepse. Lesão pulmonar aguda. Ventilação mecânica. Citocinas. Pentoxifilina.

\section{INTRODUÇÃo}

A lesão pulmonar aguda (LPA) e a síndrome do desconforto respiratório agudo (SDRA) na sepse são eventos comuns, afetando aproximadamente $43 \%$ de todos os pacientes e associada a um aumento significante na mortalidade, superior a $80 \%$ quando associada à falência de outros órgãos ${ }^{1-4}$.

U m dos pontos primários nas medidas terapêuticas na sepse severa e choque séptico é prover uma adequada oxigenação e recebimento de oxigênio ${ }^{5}$.

Vários estudos experimentais demonstraram que altos volumes correntes e pressão positiva ao final da expiração (PEEP) elevados causam uma ruptura no endotélio e epitélio pulmonar, inflamação, atelectasia, hipoxemia e liberação de mediadores inflamatórios ${ }^{6,7,8}$. Estudos clínicos demonstraram que a ventilação com baixo volume corrente pode melhorar estes índices ${ }^{9,10}$, sendo que estes achados estão bem relatados na redução da lesão por insuflação/desinsuflação pulmonar ${ }^{11,12}$, menor barotrauma ${ }^{13}$ e diminuição da produção de mediadores inflamatórios ${ }^{6,14,15}$.

$\mathrm{Na}$ literatura são referidos trabalhos que avaliaram os efeitos do suporte ventilatório na disfunção pulmonar e na resposta inflamatória sistêmica em animais expostos previamente à lesão, utilizando administração de ácido oléico ${ }^{16}$, instilação endotraqueal de ácido clorídric ${ }^{17}$, injeção endovenosa ou instilação endotraqueal de lipopolissacárides ${ }^{18}$, lavagem

1. Graduação em Fisioterapia - Gasoterapeuta na Universidade Federal de São Paulo - Escola Paulista de Medicina, São Paulo, SP

2. Doutoranda - Bióloga na Universidade Federal de São Paulo - Escola Paulista de Medicina. São Paulo, SP

3. Doutoranda - Pós-graduanda na Universidade Federal de São Paulo - Escola Paulista de Medicina. São Paulo, SP

4. Mestranda - Pós-graduanda na Universidade Federal de São Paulo - Escola Paulista de Medicina. São Paulo, SP

5. Livre-Docente - Docente na Universidade Federal de São Paulo - Escola Paulista de Medicina. São Paulo, SP

6. Especialista - Médico na Universidade Federal de São Paulo - Escola Paulista de Medicina. São Paulo, SP

7. Livre-Docente - Diretora da Graduação, Campus Diadema - UNIFESP. São Paulo, SP, Brasil

8. Pós-doutorado - Docente na Universidade Federal de São Paulo - Escola Paulista de Medicina. São Paulo, SP 
broncoalveolar com solução salina ${ }^{19}$, ligadura e perfuração cecal $^{20}$.

Em nosso meio, desenvolvemos um modelo de disfunção pulmonar, com foco infeccioso, que tem mostrado relevância clínica no estudo das doenças, tendo ainda a possibilidade de se testar intervenções terapêuticas. Nesse modelo, demonstramos que a pentoxifilina (PTX) reduz as proteínas totais no lavado broncoalveolar de animais sedados e em respiração espontânea, submetidos a infusão intraperitoneal de Escherichia coli (E. coli $)^{21}$.

0 presente estudo teve como objetivo avaliar as alterações bioquímicas e morfométricas no pulmão de ratos com disfunção pulmonar induzida por sepse e suporte ventilatório tratados com pentoxifilina.

\section{Métodos}

O protocolo experimental foi previamente aprovado pelo Comitê de Ética em Pesquisa da Universidade Federal de São Paulo e está em conformidade com o Guia para Cuidados e U so de Animais de Laboratório ${ }^{22}$

Animais: Foram utilizados 15 animais $(n=15)$ da colônia OUTB EPM-1-Wistar, de aproximadamente seis meses de idade, com peso médio de 350 gramas, provenientes do Centro de Desenvolvimento de Modelos Experimentais em Medicina e Biologia (CEDEME) da Universidade Federal de São Paulo Escola Paulista de Medicina (UNIFESP/EPM) manipulados de acordo com os princípios éticos para experimentação. No inicio foram aclimatizados ao ambiente do laboratório durante três dias antes do experimento com água e comida ad libitum. Após o período de adaptação os ratos foram divididos em três grupos: GC (Controle, $n=5$ ) - animais com infusão de solução salina e ventilados mecanicamente; GS (Sépticos, $n=5$ ) - animais com sepse induzida e mecanicamente ventilados; e GS+PTX (Sépticos+Pentoxifilina, $n=5$ ) - animais com sepse induzida e tratados com pentoxifilina.

Os animais dos grupos GS e GS+PTX receberam injeção intraperitoneal (i.p) de Escherichia coli e foram observados previamente por seis horas. Após este período foram randomizados para receberem pentoxifilina (PTX) ou solução salina $(0,9 \%)$, seguida de suporte ventilatório.

Preparação das bactérias: A concentração do inóculo e 0 tempo para o desenvolvimento da sintomatologia de sepse encontram-se previamente estabelecidos em nosso laboratório 21. Consiste na administração intraperitoneal de $1 \mathrm{~mL} / 100 \mathrm{~g}$ de peso corporal ( $1 \mathrm{~mL}$ corresponde a $10^{7}$ unidades formadoras de colônia - UFC), de uma solução de bactérias, essas bactérias foram classificadas pela bioquímica e morfologia como Escherichia coli R-6.

Procedimento operatório: Após seis horas os animais foram anestesiados i.p. com tiopental sódico (Thionembutal, ABBOTT, SP, Brasil; 40 mg/kg de peso corporal), sendo imediatamente realizada traqueostomia com uma cânula de 14-GA, a jugular cateterizada (cateter - PE-50) para a administração de drogas e a artéria carótida esquerda cateterizada (cateter - PE50) para análise da pressão parcial do oxigênio.

Gasometria arterial: A análise da pressão parcial de oxigênio $\left(\mathrm{PaO}_{2}\right)$ foi realizada com 0 auxílio de uma seringa estéril (PICO 70, Radiometer, Copenhagen, Denmark) e avaliada no aparelho AVL Compact 3 (Roche Diagnostic, Mannheim, Germany) nos tempos Zero ( $Z$; após seis horas da infusão do inóculo), 60, 120 e 180 min (após 60, 120 e 180 minutos da administração da PTX ou da solução salina seguida de ventilação mecânica, respectivamente).

Infusão de pentoxifilina ou solução salina: Após a primeira análise gasométrica (tempo zero) os animais receberam infusão de $25 \mathrm{mg} / \mathrm{kg}$ de PTX (Sigma, St. Louis, USA) ou solução salina (no mesmo volume) e, após 20 minutos, os animais foram submetidos a ventilação mecânica.

Estratégia ventilatória: Vinte minutos após a administração de PTX (ou solução salina) os animais foram paralisados com brometo de pancurônio - relaxante muscular - (Pavulon, Organon, SP, Brasil) $2 \mathrm{mg} / \mathrm{kg}$ i.p. Apenas uma estratégia ventilatória foi aplicada durante 180 minutos para todos os grupos, sendo: volume corrente $(\mathrm{VC})=6 \mathrm{~mL} / \mathrm{kg}$, freqüência respiratória (fR) 30-50 respirações/min (bpm), (PEEP) $5 \mathrm{~cm}$ $\mathrm{H}_{2} \mathrm{O}$, e fração inspirada de oxigênio [FIO2] de 1.0).

Lavado broncoalveolar (LBA): Ao final do período de 180 minutos, os animais foram submetidos a eutanásia $(1 \mathrm{~mL} / 100$ $\mathrm{g}$ de peso corporal; T-61 Euthanasia Solution, Hoechst \& Roussel, EUA), seguida de esternotomia, sendo os pulmões e estruturas mediastinais removidas e pesadas. Foi efetuado bloqueio no brônquio esquerdo e o pulmão direito lavado com solução tampão fosfato (PBS) a $4{ }^{\circ} \mathrm{C}$ por três vezes $(20 \mathrm{~mL} / \mathrm{kg}$ de peso corporal). 0 volume retornado foi centrifugado a $400 \mathrm{x}$ g por 10 minutos a uma temperatura de $4{ }^{\circ} \mathrm{C}$ e 0 sobrenadante armazenado em freezer $-80^{\circ} \mathrm{C}$.

Relação peso úmido/peso seco do pulmão (W/D): Após a lavagem broncoalveolar, os pulmões direitos foram armazenados em estufa de secagem (EB-60, Labtrade, SP, Brasil) por 72 horas a uma temperatura de $60{ }^{\circ} \mathrm{C}$ sendo novamente pesados.

Proteínas totais: Em uma alíquota de $1 \mathrm{~mL}$ do LBA foram mensuradas as proteínas totais com reagente específico (QUANT-iT P rotein Assay, Invitrogen, CA, USA) e a leitu ra realizada no equipamento QUBIT Fluorometer (Invitrogen, CA, USA).

Histologia: Os pulmões esquerdos foram inflados até uma pressão de $20 \mathrm{cmH}_{2} \mathrm{O}$ com formaldeído a $4 \%$ (PBS 0,1 mol; pH 7,4 ) em temperatura ambiente para fixação. Em seguida, foram cortados e os fragmentos fixados na mesma solução durante 12 horas. Em seguida, foram desidratados em concentrações crescentes de etanol segundo as técnicas histológicas para inclusão em parafina. Os fragmentos pulmonares foram cortados em seções de $4 \mu \mathrm{m}$ e os cortes corados pela hematoxilina e eosina (H.E.).

A espessura dos septos alveolares foi realizada utilizando-se um sistema de captura de imagem que consiste em microscópio de luz (Axiolab Standart 2.0, Carl Zeiss, Germany) acoplado a uma câmara de vídeo de alta resolução (AxionCam, Carl Zeiss, Germany) e programa específico para morfometria (Axiovision 4.2 Real, Carl Zeiss, Germany).

Análise estatística: Os dados obtidos foram expressos em tabelas e gráficos como média \pm desvio padrão ( $M \pm D P)$. Os grupos foram comparados entre si por meio de uma análise de variância (ANOVA) e, quando necessário, teste de comparações múltiplas. Todos os testes foram realizados utilizando-se programa de estatística específico (SPSS 10.0, SPSS Science, Chicago, IL, USA). Adotou-se como nível de significância $P<0,05$ (5\%). 


\section{Resultados}

Antes de se realizar a ventilação mecânica, notamos que todos os animais injetados com E. coli apresentaram piloereção, diarreia, olhos incrustados e letargia caracterizada pela redução do estado de alerta e mobilidade.

Avaliação da gasometria arterial: No grupo séptico (GS) notamos, após $6 \mathrm{~h}$ pós- inoculação da $\mathrm{E}$. coli, que a $\mathrm{PaO}_{2}$ se apresentou reduzida no tempo $Z(57,0 \pm 3,9 \mathrm{mmHg})$, em relação ao grupo GC, e aumentada nos tempos 60 e 120 minutos de ventilação mecânica $(145,0 \pm 23,7$ e 354,0 \pm $22,9 \mathrm{mmHg}$, respectivamente), contudo decresceu aos 180 minutos $(336,0 \pm 14,6 \mathrm{mmHg})$. No grupo GS+PTX, no tempo $\mathrm{Z}$, a $\mathrm{PaO}_{2}$ não diferiu do grupo GS, contudo, durante o decorrer do experimento, a análise estatística mostrou diferenças significantes entre os grupos (GS vs GS+PTX) nos tempos 60, 120 e 180 minutos (Figura 1).

Avaliação das proteínas totais: As proteínas totais no lavado broncoalveolar (Tabela 1 ) demonstraram significante aumento no grupo GS comparado ao grupo GC $(P<0.05)$ e redução no grupo GS+PTX comparado ao grupo $G S(P<0,05)$.

Avaliação do peso dos pulmões: Observou-se diferença significante na relação peso úmido/peso seco (W/D) nos animais do grupo GS comparados aos do grupo GS+PTX (Tabela 1) ao final do experimento.

Morfologia: A análise histológica mostrou, após três horas de ventilação mecânica no grupo GC, alvéolos íntegros, com septos delgados, com vasodilatação, revestidos por pneumócitos tipos I e II e lúmen alveolar vazio (Figs. 4A e B). Já no grupo GS notou-se a presença de septos pulmonares espessos, com vasodilatação e infiltração leucocitária (Fig. 4C). No interior de alguns alvéolos, identificou-se a presença de inúmeros macrófagos, leucócitos e plasma (Fig. 4D). No grupo GS+PTX, essas alterações foram mais atenuadas (Figs. 4E e F).

Morfometria: Os dados expressos na Tabela 1 mostram redução na espessura do septo alveolar no grupo GS+PTX $(11,15 \pm 3,62 \mu \mathrm{m})$, provavelmente ocasionada pela ação do PEEP sobre o alvéolo quando comparados aos do grupo GS $(19,30 \pm 3,75 \mu m)(P<0,05)$.

\section{Discussão}

Os resultados do presente estudo mostraram que a pentoxifilina promoveu substancial redução na concentração de proteínas totais no lavado broncoalveolar e na relação peso úmido/peso seco, acompanhada de um aumento na oxigenação no modelo de disfunção pulmonar induzida por infusão intraperitoneal de Escherichia coli.

A melhora pulmonar, com o uso da pentoxifilina, nos parâmetros analisados estão relacionados a possíveis bloqueios dos vários mecanismos inflamatórios desencadeados por esta substância23. A pentoxifilina, 1-(5-oxohexil) - 3,7 dimetilxantina ou 1-(5 oxohexil), teobromina ou oxipentifilina é uma metilxantina derivada da teobromina, semelhante à cafeína e à teofilina, que inibe vários mecanismos inflamatórios, incluindo a cascata do complemento, a aderência dos neutrófilos e a produção de citocinas ${ }^{24}$.

Em relação ao modelo experimental, a maioria dos estudos utiliza a ligadura e punção cecal como modelo de sepse peritoneal. Nós desenvolvemos e escolhemos a injeção ip de

\begin{abstract}
Figura 1 - Representação gráfica demonstrando os efeitos da inoculação intraperitoneal de E. coli na gasometria arterial. GC: grupo apenas ventilado; GS: grupo séptico e ventilado; GS + PTX: grupo séptico, tratado com pentoxifilina e ventilado. $* p<0,05$ vs. GS e GS+ PTX no tempo zero; ${ }^{\#} p<0,05$ vs GS no tempo 60 minutos; ${ }^{\S} p<0.05$ vs. GC e GS no tempo 120 minutos; $\mathrm{e}^{\ddagger} \mathrm{p}<0.05$ vs GC e GS+ PTX no tempo 180 minutos.
\end{abstract}

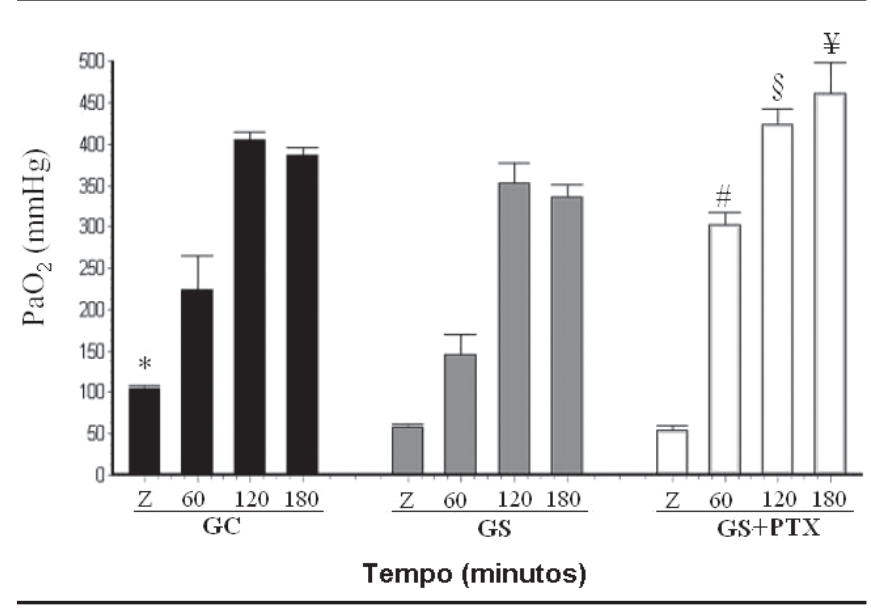

Tabela 1 - Médias e desvios padrão da dosagem das proteínas totais no lavado broncoalveolar, diâmetro dos septos alveolares e relação peso úmido/seco (W/D) dos pulmões dos animais pertencentes aos vários grupos de estudo (GC = grupo controle; GS = grupo com sepse e GS+ PTX grupo com sepse tratado com pentoxifilina)

\begin{tabular}{lccr}
\hline & \multicolumn{3}{c}{ Grupos } \\
\cline { 2 - 4 } & GC & GS & GS+PTX \\
\hline Prot. Totais $(\mathrm{mg} / \mathrm{mL})$ & $16,0 \pm 0,5$ & $21,0 \pm 1.2^{*}$ & $18,0 \pm 0,5$ \\
Sep. Alveolar $(\mu \mathrm{m})$ & $8,4 \pm 1,1$ & $19,3 \pm 3,7^{*}$ & $11,1 \pm 3,6$ \\
W/D & $6,0 \pm 0,2$ & $12,0 \pm 1,1^{*}$ & $8,0 \pm 0,9$ \\
\hline "P $<0.05$ comparado com os grupos GC e GS+PTX
\end{tabular}

E. coli tendo em mente os efeitos lesivos do procedimento operatório que, per se, desencadeia um trauma e, consequentemente, processo inflamatório ${ }^{21}$. A escolha da estratégia e parâmetros ventilatórios deu-se por ser o procedimento utilizado em unidade de cuidados intensivos e no início de alguns procedimentos cirúrgicos.

$\mathrm{N}$ a sepse, produtos tóxicos ativam as defesas sistêmicas do hospedeiro incluindo neutrófilos, macrófagos, monócitos, céIulas endoteliais e o sistema complemento ${ }^{25}$, além de levar a uma hipoxemia, sendo necessária a utilização da ventilação mecânica ${ }^{25}$.

Vários estudos mostraram que a ventilação com baixo volume corrente reduz relativamente o risco de mortalidade em pacientes com a síndrome do desconforto respiratório agudo ${ }^{9-}$ 15,26,27. Sob condições experimentais, a sepse pode ser estimulada por administração de bactérias ou produtos bacterianos (endotoxinas). Durante a sepse, alterações estruturais ocorrem nos capilares das células endoteliais que aumentam a permeabilidade dos capilares ocasionando edema ${ }^{21}$. Em nosso 


\begin{abstract}
Figura 2 - Fotomicrografias mostrando parênquima pulmonar de ratos pertencentes ao vários grupos de estudo. A e B grupo controle, observar alvéolos íntegros, as setas indicam os septos alveolares. C e D grupo com sepse, observar parte dos alvéolos repletos de macrófagos (setas) e E e F grupo com sepse tratado com pentoxifilina, observar maior concentração de alvéolos contendo ar no seu interior e septos alveolares congestos. H.E. A, C e E 100X; B, D e F 400X.
\end{abstract}

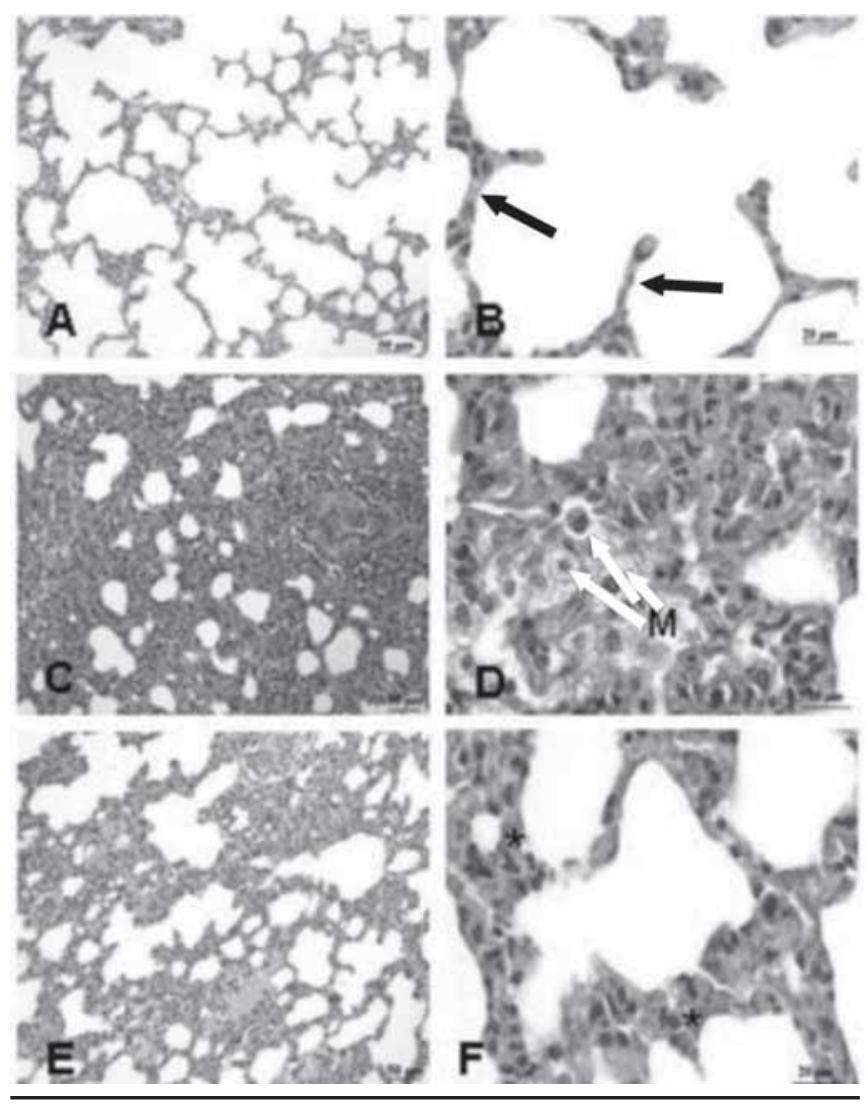

modelo experimental, notamos infiltrado linfoplasmocitário nos alvéolos dos animais induzidos pelo sepse e aumento nas concentrações de proteínas totais no lavado broncoalveolar. Porém, este último achado foi reduzido após administração de pentoxifilina.

É relatado que no septo alveolar, no qual um sistema de fibras axiais e periféricas está interconectado, ocorre um aumento das fibras. Isso ocorre por um excesso de tensão nas estruturas pulmonares, por minutos ou dias, dependendo da espécie animal, causa edema pulmonar de graus variados associado a prejudicial troca gasosa, formação de membrana hialina e infiltração de neutrófilos e de macrófagos ${ }^{27}$. Em nosso material, percebemos aumento na espessura dos septos, que acreditamos ser devido à intensa vasodilatação.

Nossos resultados mostraram que existe um grande comprometimento dos septos alveolares nos animais com disfunção pulmonar induzida pela administração de bactérias (Escherichia coli), porém tais valores se alteram, ficando mais parecidos com o grupo normal, quando os animais com disfunção são tratados com PTX. Neste último grupo notamos alvéolos e septos pulmonares muito parecidos aos dos animais do grupo controle.

Mercer et al. demonstraram que mesmo volumes correntes normalizados induzem um aumento no diâmetro alveolar de $40 \%{ }^{28}$. Estudos de Creamer et al. mostraram que a PTX previne a deterioração da complacência pulmonar e preserva a integridade vascular ${ }^{29}$. Nossos resultados corroboram esta positiva influência da PTX na estrutura alveolar.

Nosso modelo mostrou ser uma ferramenta útil para avaliar os efeitos de dois eventos, ou seja, infecção pela Escherichia coli seguida de ventilação mecânica, uma vez que estímulos gerados nesta associação diferem daqueles desencadeados apenas pela injeção de E. coli e a possibilidade de intervenções terapêuticas torna-se uma realidade. Em conclusão, a PTX demonstrou eficiência na redução do dano pulmonar neste modelo experimental.

\section{Agradecimentos}

Ao Prof. Dr. Ivan H. Koh, Ana M. A. Liberatore e MarjorieY. Taki pelas bactérias utilizadas.

\section{Conflito de interesse: não há}

\section{SUMMARY}

HISTOMORPHOLOGIC AND RESPIRATORY ASPECTS OF ACUTE LUNG INJURY IN RATS INDUCED BY EXPERIMENTAL SEPSIS AND UNDER PENTOXIFYLLINE TREATMENT

Oвjective. Respiratory repercussion on acute lung injury in a model of induced sepsis intraperitoneally.

METHODS. Fifteen animals taken at random were submitted to adult male $\mathrm{W}$ istar rats. The rats were randomly divided into 3 groups $(n=15)$ : Group C - control group received only mechanical ventilation; Group S - rats received live Escherichia coli (E. coli) intraperitoneally (septic) and after 6 hours they were treated with normal saline infusion and ventilated with a low tidal volume. Group S+ PTX - rats received live Escherichia coli intraperitoneally (septic) and after 6 hours they were treated with pentoxifylline (PTX) infusion and ventilated with a low tidal volume. All animals were ventilated during 180 minutes. We analyzed the arterial blood gases, gravimetric indices and histomorphometric analysis.

RESULTS. Blood gases, wet to dry ratios, and total protein concentrations in the bronchoalveolar lavage were analyzed in all experimental groups. In the end of the experiment the partial pressure of oxygen was higher in the GS+PTX $(460,0 \pm 38,2$ $\mathrm{mmHg})$ compared with GS $(336,0 \pm 14,6 \mathrm{mmHg})$. Pentoxifylline with low tidal volume attenuated significantly total protein in the bronchoalveolar lavage. The septal diameter was significantly reduced in the group GS compared with group GS+PTX $(P<0,05)$.

Conclusions. The pentoxifylline ameliorated the oxygenation and decreased the deleterious effects of sepsis in the associated mechanical ventilation. [Rev Assoc Med Bras 2009; 55(2): 127-31]

Key words: Sepsis. Acute lung injury. Mechanical ventilation. Cytokine. Pentoxifylline. 


\section{REFERÊNCIAS}

1. Fein AM, Lippman M, Holtzman H, Eliraz A, Goldberg SK. The risk factors, incidence and prognosis of ARDS following septicemia. Chest. 1983;83:40-2.

2. Hudson LD, Milberg J A, Anardi D, Maunder RJ. Clinical risk for development of the acute respiratory distress syndrome. Am J Respir Crit Care Med. 1995;151:293-301.

3. Mathay MA. Conference summary: acute lung injury. Chest. 1999;116: 119S-26S.

4. Morrison RJ, Bidani A. Acute respiratory distress syndrome epidemiology and pathophysiology. Chest Surg Clin North Am. 2002;12:301-23.

5. Balk RA. Optimum treatment of severe sepsis and septic shock: evidence in support of the recommendations. Disease-a-M onth. 2004; 50: 168-213.

6. Tremblay L, Valenza F, Ribeiro SP, Li J, Slutsky AS. Injurious ventilatory strategies increase cytokines and c-fos $\mathrm{m}$-RNA expression in an isolated rat lung model. J Clin Invest. 1997;99:944-52.

7. Parker J C, Hernandez LA, Peevy KJ. Mechanisms of ventilator-induced lung injury. Crit Care Med. 1993;21:131-43.

8. Webb HH, Tierney DF. Experimental pulmonary edema due to intermittent positive pressures ventilation with high inflation pressures. Am Rev Respir Dis. 1974:110:556-65.

9. Amato MB, Barbas CS, Medeiros DM, Magaldi RB, Schettino GP, LorenziFilho $G$, et al. Effect of a protective-ventilation strategy on mortality in the acute respiratory distress syndrome. N Engl| Med. 1998;338:347-54.

10. Acute Respiratory Distress Syndrome Network. Ventilation with lower tidal volumes as compared with traditional tidal volumes for acute lung injury and the acute respiratory distress syndrome. N Engl J Med. 2000;342:1301-8.

11. Hickling KG, Henderson SJ , J ackson R. Low mortality associated with low volume pressure limited ventilation with permissive hypercapnia in severe adult respiratory distress syndrome. Intens Care Med. 1990;16:372-7.

12. Hickling KG, Walsh S, Henderson SJ , Jackson R. Low mortality rate in adult respiratory distress syndrome using low-volume, pressure-limited ventilation with permissive hypercapnia: a prospective study. Crit Care Med. $1994 ; 22: 1568-78$.

13. Dos Santos CC Slutsky AS. Invited review: mechanisms of ventilatorinduced lung injury: a perspective. J Appl Physiol. 2000;89:1645-55.

14. Parsons PE, Eisner MD, Thompson BT, Matthay MA, Ancukiewicz M, Bernard GR, et al. Lower tidal volume ventilation and plasma cytokine markers of inflammation in patients with acute lung injury. Crit Care Med. 2005;33:1-6.

15. Ricard J C, Dreyfuss D, Saumon G. Production of inflammatory cytokines in ventilator-induced lung injury: a reappraisal. Am J Respir Crit Care Med. 2001;163:1176-80

16. DiRocco J D, Pavone LA, Carney DE, Lutz CJ, Gatto LA, Landas SK, et al. Dynamic alveolar mechanics in four models of lung injury. Intensive Care Med. 2006;32:140-8
17. Gurkan OU, O'Donnell C, Brower R, Ruckdeschel E, Becker PM. Differential effects of mechanical ventilation strategy on lung injury and systemic organ inflammation in mice. Am J Physiol Lung Cell Mol Physiol. 2003;285:L710 L8.

18. Wischmeyer PE, Kahana M, Wolfson R, Ren $H$, Musch MM, Chang EB. Glutamine reduces cytokine release, organ damage, and mortality in a rat model of endotoxemia. Shock. 2001;16:398-402.

19. Murphy DB, Cregg N, Tremblay L, Engelberts D, Laffey J G, Slutsky AS, et al. Adverse ventilatory strategy causes pulmonary-to-systemic translocation of endotoxin. Am J Respir Crit Care Med. 2000;162:27-33.

20. Rasaiah VP, Malloy J L, Lewis JF, Veldhuizen RA. Early surfactant administration protects against lung dysfunction in a mouse model of ARDS Am J Physiol Lung Cell Mol Physiol. 2003;284:L783-L90.

21. Oliveira-J únior IS, Brunialti MKC, Koh IHJ, J unqueira VBC, Salomão R Effect of pentoxifylline on lung inflammation and gas exchange in a sepsis-induced acute lung injury model. Braz J Med Biol Res 2006;39:1455-65

22. Olfert ED, Cross BM, McWilliam AA, editors. Canadian Council on Animal Care's/Guide to the Care and Use of experimental Animals. $2^{\text {nd }}$ ed. Ottawa: Bradda Printing Services; 1993.

23. Oliveira-J unior IS, Pinheiro BV, Silva ID, Salomao R, Zollner RL, Beppu OS. Pentoxifylline decreases tumor necrosis factor and interleukin-1 during high tidal volume. Braz J Med Biol Res. 2003;36:1349-57.

24. Iskesen I, Saribulbul O, Cerrahoglu M, Onur E, Destan B, Sirin BH. Pentoxifylline affects cytokine reaction in cardiopulmonary bypass. Heart Surg Forum. 2006;9:E883-7.

25. Natanson C, Hoffman WD, Suffredini AF, Eichacker P, Danner RL. Selected treatment strategies for septic shock based on proposed mechanisms of pathogenesis. Ann Intern Med. 1994;120:771-83.

26. Ware LB and Matthay MA. The acute respiratory distress syndrome. N Engl Med. 2000:342:1334-48.

27. Gattinoni L, Carlesso E, Cadringher P, Valenza F, Vagginelli F, Chiumello D. Physical and biological triggers of ventilator-induced lung injury and its prevention. Eur Respir J. 2003;22:15S-22S.

28. Mercer RR, Russell ML, Crapo JD. Alveolar septal structure in different species. J Appl Physiol. 1994;77:1060-6.

29. Creamer KM, McCloud LL, Fisher LE, Ehrhart IC. Pentoxifylline rescue preserves lung function in isolated canine injured with phorbol myristate acetate. Chest. 2001;199:1893-1900.

Artigo recebido: 06/11/07

Aceito para publicação: 02/06/08 